
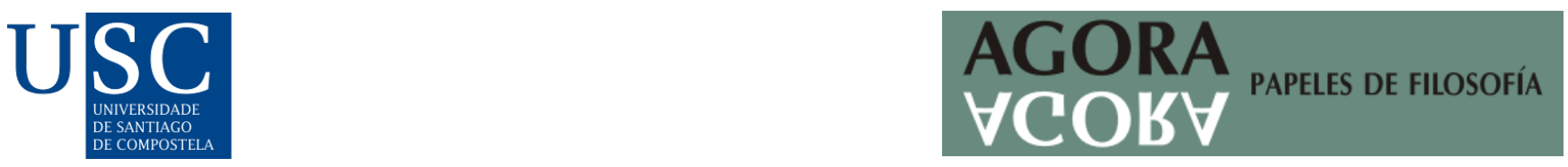

Agora. Papeles de Filosofía, 41(1), 2022. ISSN: 2174-3347

https://doi.org/10.15304/agora.41.1.7416

Estudios

\title{
UNA DEFENSA DE LAS AXIOLOGÍAS CENTRADAS EN EL SUFRIMIENTO
}

\author{
Amanda Briones Marrero ${ }^{1}$ iD \\ ${ }^{1}$ Universidad de Oviedo, España
}

Recibido: 19/01/2021; Aceptado: 09/03/2021

\section{Resumen}

Este artículo realiza una defensa de las axiologías centradas en el sufrimiento, que sostienen la prioridad de evitar el sufrimiento frente a la promoción de otros valores. Para ello, explica en primer lugar cómo ciertas cuestiones relativas a la creación de individuos sugieren la existencia de una asimetría entre el sufrimiento y la felicidad. Continúa describiendo un conjunto de argumentos a favor de la prioridad del sufrimiento relativos a individuos ya existentes. Asimismo, señala y responde a distintas objeciones que podrían ser dirigidas contra estas axiologías. Finalmente, concluye que las posiciones centradas en el sufrimiento no están todavía lo bastante exploradas y pueden tener un gran potencial.

Palabras clave: axiología; valor; sufrimiento; asimetría

\begin{abstract}
This article makes a defence of the suffering-focused axiologies, which sustain the priority of avoiding suffering over the promotion of other values. To this end, it first explains how certain questions concerning the creation of individuals suggest the existence of an asymmetry between suffering and happiness. It goes on to describe a set of arguments in favour of the priority of suffering relative to existing individuals. It also points out and responds to various objections that could be directed against these axiologies. Finally, it concludes that suffering-focused positions are not yet sufficiently explored and may have great potential.
\end{abstract}

Keywords: axiology; value; suffering; asymmetry

\section{INTRODUCCIÓN}

Muchas personas tienen la intuición de que la reducción del sufrimiento tiene más peso que la promoción del disfrute o de otros valores positivos. Como consecuencia, esta idea ha aparecido de manera más o menos esporádica en la literatura filosófica. Sin embargo, solo en tiempos muy recientes ha sido formulada de manera explícita para proporcionar la base de una posición moral claramente diferenciada. Se ha empleado el término omniabarcante "éticas centradas en el sufrimiento" para englobar a todas aquellas teorías éticas que aceptan, formulada de una manera u 
otra, tal posición. Estas éticas pueden tener una versión normativa, cuando se defienden porque se considera que deberíamos dar prioridad a la reducción del sufrimiento. 0 pueden tener una versión axiológica, cuando se defienden considerando que es peor que haya sufrimiento a que no haya valores positivos como el placer. Este artículo se centrará en examinar los modos en los que pueden defenderse las posiciones de este último tipo, esto es, las axiologías centradas en el sufrimiento.

Para defender tales axiologías, el primer apartado se centrará en exponer la asimetría que existe entre la felicidad y el sufrimiento, asimetría que se hace patente en los planteamientos relativos a la creación de nuevos individuos. El segundo apartado, por su parte, se focalizará en una serie de argumentos que apoyan la afirmación de que el sufrimiento es prioritario considerando a los individuos que ya existen. Por último, el tercer apartado pretenderá responder a las posibles objeciones que podrían ser dirigidas contra estas axiologías.

\section{LA ASIMETRÍA ENTRE EL SUFRIMIENTO Y LA FELICIDAD EN LA CREACIÓN DE INDIVIDUOS}

Muchas personas tienen la intuición de que existe una asimetría entre traer a la vida a seres cuyas existencias van a ser felices o desgraciadas (Narveson, 1978; Tooley, 1998; Elstein, 2005; Roberts, 2011a; Roberts, 2011b; Algander, 2012; Meacham, 2012; Frick, 2014; Grill, 2017). Consideremos el siguiente experimento mental para ilustrar esta asimetría:

La asimetría entre traer vidas positivas y negativas. Una mujer que es feliz tiene que decidir si quedarse embarazada y dar a luz. Podemos pronosticar que, si lo hace, su felicidad disminuirá, aunque seguirá estando claramente por encima del nivel al que la vida deja de merecer la pena ser vivida. Pero también podemos pronosticar que, si lo hace, su hija o hijo será feliz.

Otra mujer, también feliz, tiene que decidir si quedarse embarazada y dar a luz. Podemos pronosticar que, si lo hace, su felicidad aumentará. Pero también podemos pronosticar que, si lo hace, su hija o hijo será muy infeliz, sufriendo mucho y teniendo una vida claramente por debajo del nivel al que merece la pena ser vivida.

La intuición de que en el primer caso sería mejor que la mujer no diese a luz se encuentra muy extendida. Tal intuición descansa en la idea de que los intereses de la mujer que existe en el presente tienen prioridad por encima de los de su posible hija futura a la hora de decir qué situación es mejor. En ese caso, entonces, si el disfrute y el sufrimiento son simétricos, deberíamos aceptar que en el segundo caso sería mejor que la mujer diese a luz, pues hemos aceptado que los intereses de la madre tienen prioridad. Si, en cambio, consideramos que en el segundo caso será necesariamente peor que la madre dé a luz, entonces, de nuevo, si disfrute y sufrimiento son simétricos, habríamos de aceptar que en el primer caso la mujer debe dar a luz.

Podemos, ante esto, rechazar guiarnos por principios, y optar simplemente por lo que nos parece

más intuitivo en cada caso. Pero esta respuesta es ad hoc, pues no proporciona una explicación de la justificación que puede llevar a tomar cualquiera de las decisiones en un caso u otro.

Frente a estas opciones no satisfactorias, una vía consistente y no ad hoc de evitar las implicaciones contraintuitivas en estos dos casos puede ser la de asumir la prioridad de evitar el sufrimiento frente a la promoción del bienestar positivo (Gardner, 2015a; Gardner, 2015b). Así, las axiologías centradas en la reducción del sufrimiento pueden proporcionar una respuesta satisfactoria ante los dos casos de este experimento mental porque, en primer lugar contemplan la no-existencia de la hija de la primera mujer como una opción al mismo nivel de valor que 
la existencia carente de sufrimiento, mientras que consideran la no-existencia de la hija de la segunda mujer mejor que su existencia padeciendo sufrimiento. ${ }^{1}$ Por tanto, quien defienda estas axiologías podría responder justificadamente que es preferible que la mujer no tenga descendencia en ninguno de los dos casos, aunque en el primero se esté pensando en sus intereses, y en el segundo en los intereses de la posible hija.

Si aceptamos la prioridad del sufrimiento para solventar el problema al que nos enfrenta La asimetría entre traer vidas positivas y negativas, estaríamos asumiendo que existe, pues, una asimetría entre el sufrimiento y la felicidad (Mayerfeld, 1996; Hurka, 2010). Esta asimetría puede ser defendida también de otros modos que veremos a continuación.

\section{ARGUMENTOS A FAVOR DE LA PRIORIDAD DEL SUFRIMIENTO CONSIDERANDO A LOS INDIVIDUOS QUE YA EXISTEN}

Las intuiciones a favor de las axiologías centradas en el sufrimiento pueden defenderse también de maneras que no apelan a la creación de nuevos individuos. Para ello, podemos considerar nuestras intuiciones en diferentes casos en los que comparamos el valor del sufrimiento con el del bienestar positivo, incluso cuando este último consiste en experiencias de disfrute iguales en duración e intensidad a las del sufrimiento considerado. Algunos de los principales argumentos que podemos considerar aquí son los siguientes:

Asimetría en la importancia de los distintos niveles de sufrimiento y felicidad. La mayoría entendemos que no resulta moralmente urgente convertir los estados de satisfacción o tranquilidad en estados de máximo placer. Existen experiencias aparentemente neutrales que, a pesar de no proporcionar picos de placer intenso, tampoco contienen experiencias negativas, resultando por ello neutrales o ligeramente positivas. Tal es el caso de los estados meditativos profundos, por ejemplo. Normalmente consideramos que no hay una gran pérdida de valor en el hecho de que alguien se encuentre en tal estado de tranquilidad en lugar de en un estado de placer extremo (Gloor, 2017; Gloor, 2019).

Consideremos, sin embargo, una situación inversa. Supongamos que alguien se encuentra en estado de sufrimiento extremo, semejante en intensidad al placer extremo en el caso anterior. Imaginemos que se pudiese cambiar su situación a otra neutral o ligeramente negativa (por ejemplo, una situación de tranquilidad solamente alterada por una levísima molestia). Consideraríamos que, en ese caso, la pérdida de valor de no hacer este cambio sí sería de enorme importancia.

Así, si tuviésemos que escoger solo una de entre dos opciones, siendo la primera favorecer que alguien cuya situación es neutral obtenga un gran placer, y siendo la segunda mitigar el sufrimiento de otro individuo, pasándolo de extremo a moderado, muchas personas entenderían que ayudar al segundo individuo causaría una mejora general en la situación mucho mayor que ayudar al primero, dado que evitar un sufrimiento extremo parece prioritario con respecto a aumentar la felicidad (Ryder, 1999; Ryder, 2005). Sin embargo, si aceptásemos la simetría del placer y el sufrimiento, ambas opciones deberían resultar equivalentes. Esto sugiere la existencia de una asimetría entre los distintos niveles de las experiencias positivas y negativas. ${ }^{2}$ Según ésta, el valor de la diferencia entre estados positivos de distinta intensidad es menor que la existente entre estados negativos de distinta intensidad. Si aceptamos esto, hemos de concluir que las experiencias negativas contienen 
más valor negativo que las experiencias positivas de igual intensidad (permaneciendo el resto de las cosas, como la duración de las experiencias y los otros efectos que estas puedan tener, iguales).

Asimetría comparativa en el nivel interpersonal. Muchas personas entienden que, de tener que elegir ante una determinada situación, es mejor abstenerse de actuar antes que torturar a alguien y causar placer a otro individuo. Aceptar un sufrimiento en el caso personal no equivale a reconocer que, en general, la felicidad pueda ser mayor que el sufrimiento al nivel de la tortura en las compensaciones interpersonales (Gloor, 2019). Podríamos tener la disposición de aceptar en nuestras vidas un sufrimiento notable a cambio de recibir un gran placer. Con todo, la mayoría no tenemos la disposición para aceptar algo así si quien recibe el dolor y quien el placer son individuos diferentes. Dado que en el mundo el sufrimiento está distribuido entre individuos diferentes, esto podría llevarnos a concluir que la importancia del sufrimiento es mayor de la que seríamos conscientes si solo tuviésemos en cuenta lo que experimentamos en nuestras propias vidas.

La preponderancia del sufrimiento extremo. Muchas de las personas que han sido sometidas a una tortura extrema son incapaces de resistir en cierto punto, y aceptan hacer cosas que jamás habrían pensado hacer, como, por ejemplo, delatar a seres queridos u otros inocentes, dar información crítica contra causas en las que creen profundamente o mediante confesiones privarse a sí mismas de la libertad o hasta la vida. Quienes resisten la tortura y evitan confesar, lo consiguen solamente con un esfuerzo extremo. Sin embargo, muchas de esas personas evitarían hacer eso a cambio de cualquier cantidad de placer sin ni siquiera plantearse la cuestión. Esto sugiere que el desvalor del sufrimiento es realmente mayor que el valor positivo del placer.

En conjunto, estos tres argumentos nos dan razones a favor de aceptar el desvalor especial del sufrimiento. Pero hay otro tipo de razones a favor de esta posición que podemos tener en cuenta también que no apelarían a cuestiones axiológicas, sino fácticas (Vinding, 2020). Serían relativas al modo en el que tiene lugar el sufrimiento en el mundo, y a la cantidad de éste que existe. Estas pueden complementar los argumentos axiológicos que vimos arriba porque, de ser correcto lo que indican, vendrían a implicar que la asunción de axiologías centradas en el sufrimiento podría tener las mejores consecuencias desde el punto de vista del nivel de valor esperable. Esto sería así incluso aunque los argumentos indicados arriba no fueran correctos. Estas consideraciones fácticas serían las siguientes:

La tolerancia hacia la felicidad. En general, parece que los individuos tienden a generar una cierta tolerancia hacia la felicidad, mas no sucede así con situaciones de padecimiento físico y/o mental (Mayerfeld, 1999, p. 39; Bratslavsky et al., 2001). Comúnmente las personas se ven afectadas por acontecimientos positivos en sus vidas de manera solo temporal, pues tienen tendencia a regresar a un estado de neutralidad hedónica. ${ }^{3}$ Esto puede implicar que intentar aumentar la felicidad podría ser poco útil si, a medida que se incrementan los factores que constituyen el bienestar de un individuo, también crecen sus expectativas y deseos, con lo que no se produciría, en último término, un aumento permanente de la felicidad, sino una cierta tolerancia hacia ella (Diener et al., 2006).

Asimetría cuantitativa. Muchas personas entienden que, tanto en el pasado como en la actualidad, ha habido más cantidad de sufrimiento que de felicidad (Mayerfeld, 1999, p. 67; Mathison, 2018, pp. 6-23; Smith, 1759, p. 44). Este argumento goza de cierta popularidad por haber sido defendido por Schopenhauer (1989; 1999; 2005). Según el pesimismo schopenhaueriano, la existencia de todo individuo está inevitablemente llena de sufrimiento. Todo individuo tiene voluntad de querer, pero la mayoría de sus deseos se ven finalmente insatisfechos, lo cual le supone un continuo estado de esfuerzo y privación. Es más: cuando alguien consigue satisfacer un anhelo, 
esta satisfacción es solo aparente, pues cada deseo cumplido deja paso, rápidamente, a uno nuevo. Así, la felicidad puede considerarse un mero cese temporal de algún sufrimiento particular ${ }^{4}$.

Siguiendo esta idea, este argumento sostiene que incluso si considerásemos que, de manera particular, numerosos individuos tienen o han tenido más felicidad que sufrimiento en sus vidas, hay razones de peso para concluir que la cantidad de sufrimiento en el mundo prevalece sobre la cantidad de felicidad. ${ }^{5}$ En el caso humano existen muchas causas de sufrimiento a gran escala, como las guerras, los genocidios, el hambre, la esclavitud o la multiplicidad de enfermedades que existen (Singer, 2010; Unger, 1996). Puede ser que el sufrimiento ocasionado por estas sea menor que el disfrute humano pero, incluso si es así, la balanza puede verse inclinada hacia el otro lado por el sufrimiento de los animales no humanos, que ha de ser considerado con la misma prioridad dada su capacidad de sintiencia y, por tanto, de sufrir (Singer, 1995). En línea no utilitarista, el dolorismo o painismo considera que el principal valor que ha de ser tenido en cuenta es la experiencia de dolor de cada individuo con independencia de la especie, entendiendo el dolor en sentido amplio, esto es, como sufrimiento tanto físico como mental (Ryder, 1992; Ryder, 1999; Ryder, 2003). Cada año una cifra de animales que podría acercarse a los 200.000 millones sufre en granjas industriales (Singer, 1995; Mood y Brooke, 2012; FAO, 2020). Además, no solo las granjas industriales son causa de terrible padecimiento: aunque la naturaleza sea mostrada comúnmente como idílica, los animales sufren enormemente en ella. La principal causa de sufrimiento en los animales salvajes se debe a la estrategia reproductiva que prevalece en la naturaleza, consistente en tener enormes cantidades de descendientes, de los cuales la gran mayoría muere poco después de su llegada a la existencia. Esto significa que la mayoría de los animales en la naturaleza tienen poco tiempo para disfrutar de experiencias positivas y a menudo mueren de maneras que implican un sufrimiento significativo, como inanición, deshidratación, frío o depredación. La dinámica de la población sugiere, pues, que el sufrimiento prevalece sobre la felicidad en la naturaleza (Faria y Paez, 2015; Faria, 2016; Tomasik, 2015; Horta, 2017; Animal Ethics, 2020).

La dificultad de evitar el sufrimiento. Muchas personas podrían entender la disminución del sufrimiento como prioritaria debido a la idea de que los individuos, en general, tienen que luchar mucho para evitarlo, mas no han de esforzarse para evitar la felicidad (en todo caso, sí para conseguirla) (Vinding, 2020, p. 28). Si una persona deja de comer (o si no lo hace equilibradamente), si no practica deporte o si no disfruta de una vida intelectual, social y tiempo de ocio que la satisfaga, puede enfermar física y mentalmente. A esto se puede añadir que parece más sencillo fracasar que tener éxito, con lo que se precisa mucho trabajo para evitar lo primero y obtener lo segundo. En el caso de los animales no humanos, especialmente en la naturaleza, sucede también que han de evitar numerosos peligros para no sufrir o simplemente para sobrevivir, como señalé anteriormente.

Facilidad con la que el sufrimiento puede tener lugar. Finalmente, el día a día de muchos individuos puede llevarlos a sostener que es más probable que cualquier estado de cosas contenga sufrimiento, mas no sucede lo mismo con la felicidad. Según esta perspectiva, un pequeño evento podría llegar a causar un gran sufrimiento de manera más fácil que a la inversa (Mathison, 2018, pp. 1-5). Por ejemplo, un pequeño descuido al volante puede culminar en una vida de dolor crónico y/o secuelas físicas (y mentales) permanentes. Asimismo, mientras que existen el dolor crónico y la depresión crónica, no existe el placer o el éxtasis crónico.

Si estos argumentos que apelan a cuestiones fácticas son correctos, sugerirían que existen motivos para considerar que prevenir el sufrimiento presente que hay en el mundo y el que podría llegar a haber en el futuro tiene prioridad sobre promover el disfrute. 


\section{OBJECIONES CONTRA LA PRIORIDAD DEL SUFRIMIENTO}

Las principales objeciones que pueden hacerse en contra de la prioridad del sufrimiento pueden clasificarse en dos tipos distintos. La mayoría de estas objeciones buscan salvar la idea de la simetría entre el sufrimiento y el disfrute (Minsky, 2006, pp. 67-68) u otros valores positivos. Sin embargo, también ha sido cuestionado el propio hecho de que tenga sentido hablar de asimetría entre la felicidad y el sufrimiento, sosteniendo que no es posible determinar si hay o no simetría. Veremos varios ejemplos de ambos tipos de objeciones a continuación, así como algunas de las respuestas que se podrían dar a estas.

Las principales defensas de la simetría axiológica del sufrimiento y el bienestar positivo son las siguientes:

La apelación a la simetría en el nivel intrapersonal cuando no hay sufrimientos extremos implicados. Arriba he argumentado que mucha gente considera que el sufrimiento tiene prioridad sobre la felicidad en el plano interpersonal. También hemos visto un argumento que sostiene que el sufrimiento extremo tiene prioridad en el caso intrapersonal. Sin embargo, podríamos considerar que, en el ámbito intrapersonal, si hablamos de sufrimientos no extremos puede que no se apliquen las mismas consideraciones que en el plano interpersonal. Esto es, aunque mucha gente rechaza sufrir una tortura terrible a cambio de recibir una gran cantidad de placer, sí que podrían aceptar, en cambio, recibir un sufrimiento moderado a cambio de un disfrute suficientemente notable, u otros valores positivos. No tenemos la disposición para aceptar algo así si quien recibe el sufrimiento y quien recibe los valores positivos son individuos diferentes, pero sí si es el mismo individuo quien voluntariamente aprueba recibir ambas experiencias. Podemos, así, pensar que nuestra evaluación de lo que sucede en el plano interpersonal está sesgada y que debemos guiarnos mejor por lo que consideramos en el intrapersonal.

Sin embargo, no está claro que sean nuestras intuiciones hacia el plano intrapersonal las que tengan que tener aquí mayor autoridad. Si sostenemos esto, podremos indicar que, en realidad, muchas de las asunciones de sacrificios en ciertos momentos vitales a favor de beneficios en otros podrían ser problemáticas (esto puede ser aceptado especialmente, aunque no solamente, por quienes tengan intuiciones que cuestionen la fuerza de la continuidad de la identidad personal a lo largo del tiempo).

Además, esta objeción no pondría en cuestión aquellas intuiciones de las axiologías centradas en el sufrimiento relativas a sufrimientos extremos, a nivel de tortura. Incluso si diésemos por válida la idea de que algunas personas aceptarían sufrir leve o moderadamente a cambio de obtener y disfrutar de algo que fuese lo bastante positivo, la mayoría de las personas rechazarían intercambiar una tortura extrema aun por una ingente cantidad de placer (Gloor, 2019).

La importancia del sufrimiento solo cuando se padece. Otra posible objeción contra la asimetría apuntaría a que los individuos solo se preocupan por reducir el sufrimiento cuando lo están experimentando (Vinding, 2020). En los momentos en los que no están sufriendo, valoran otros elementos distintos. Según esta objeción, por tanto, no habría razón para darle preponderancia al sufrimiento de manera general, pues solo es urgente eliminarlo en el momento en el que se padece.

En primer lugar, se puede argumentar que existen axiologías pluralistas que, aunque centradas en el sufrimiento, señalan la relevancia de otros valores. Tal puede ser el caso de una teoría de la lista objetiva que, aunque considere prioritaria la reducción del sufrimiento, valore también los logros o la adquisición de conocimiento. 
Además, en general, muchos individuos sostienen que el sufrimiento es muy importante e incluso, prioritario, aunque no estén necesariamente padeciéndolo. Un estudio de más de 14.000 participantes llevado a cabo por el Future of Life Institute (2017) mostró que el principal objetivo que estos contemplaban para el futuro tenía que ver con la reducción del sufrimiento, con amplio margen por encima del objetivo de maximizar las experiencias positivas. Parece improbable que la mayoría de los encuestados estuviesen padeciendo un gran sufrimiento al dar su respuesta durante el estudio (Vinding, 2020, p. 151).

La apelación al sesgo de negatividad. Hay quien podría considerar que la razón por la cual puede resultar relevante evitar o reducir el sufrimiento es fruto de una limitación epistémica, esto es, de un sesgo de negatividad (Rozin y Royzman, 2001) según el cual los eventos de naturaleza negativa suelen tener un mayor impacto en quienes los sufren que los sucesos de naturaleza positiva (Baumeister et al., 2001). Sin embargo, esta no parece una explicación completa de lo que sucede en algunos de los casos presentados arriba. El que en una situación de tortura extrema haya quienes expongan a sus seres queridos para dejar de sufrir parece difícil de explicar apelando únicamente a la influencia de un sesgo de negatividad.

Asimismo, existe también un sesgo cognitivo opuesto al de negatividad, el pensamiento desiderativo. La mayoría de las personas tiende a ajustar sus valoraciones sobre el futuro a lo que desearían que sucediese, y no a lo que más probablemente podría suceder (Bastardi et al., 2011). El efecto de este sesgo puede distorsionar nuestros juicios de manera que, al contrario de lo que sugiere la objeción, conduzca a restar importancia a la necesidad de reducir el sufrimiento.

El desvalor comparado de los sufrimientos de corta duración. A algunas personas les podría parecer contraintuitiva la idea de que un sufrimiento extremo de muy corta duración (por ejemplo, que dure un solo segundo) no pueda ser compensado por una enorme cantidad de felicidad intensa. Y, sin embargo, parece que una posición que dé prioridad al sufrimiento tendría que aceptar esto.

Ante esto, sin embargo, puede indicarse que diferentes axiologías centradas en el sufrimiento darán respuestas distintas. Quienes sostengan que todo sufrimiento tiene una prioridad léxica con respecto a la felicidad u otros valores positivos considerarán que, aunque se trate de tan solo un segundo de sufrimiento extremo, este no puede ser compensado (Mayerfeld, 1999, pp. 179-180). Esto es independiente de la cantidad de felicidad que pueda haber en un estado de cosas: si en un escenario hay una cantidad de felicidad muy alta más una cantidad de sufrimiento muy moderada, esa cantidad de sufrimiento prevalecerá sobre la enorme felicidad. También llegarán a esta conclusión quienes sostengan que el único valor existente es el desvalor. Sin embargo, quienes defiendan una priorización ponderada del sufrimiento pueden llegar a una conclusión diferente. Conforme a esta posición, puede haber una determinada cantidad de valor positiva que pueda llegar a tener la magnitud necesaria para prevalecer sobre un sufrimiento. De este modo, un sufrimiento de muy corta duración podrá verse superado en importancia por una felicidad con una duración suficientemente mayor. Por último, hay axiologías centradas en el sufrimiento que no dan prioridad a todos los sufrimientos, sino solamente a aquellos que tienen una magnitud mínimamente notable. Conforme a estas concepciones se puede concluir que un gran disfrute puede tener un valor que prevalezca sobre una ligera molestia, pero no sobre un sufrimiento de una dimensión mayor. Estas axiologías pueden implicar que un solo segundo no es suficiente para que un sufrimiento, aunque sea extremo, tenga una prioridad del mismo tipo que tendría si su duración lo hiciese una experiencia más significativa, como sí podría suceder si durase unos minutos. 
Las objeciones vistas arriba tienen todas ellas como objeto defender la simetría. La otra forma de cuestionar la asimetría, que pasa por cuestionar la posibilidad epistemológica de identificar la existencia de su presencia, e incluso el sentido de hablar de esta, puede presentarse como sigue:

La subjetividad de la medida de las experiencias positivas y negativas. Algunas personas podrían apelar a la dificultad de medir el valor del sufrimiento y el bienestar positivo de manera objetiva (Giordano et al., 2010; Derbyshire, 2016). Por este motivo, hay quien podría sostener que no cabe hablar ni de simetría ni de asimetría en términos objetivos, pues la evaluación de estos depende de la percepción subjetiva de cada individuo. ${ }^{6}$

La medición subjetiva, por su parte, tampoco parece ser una solución satisfactoria a esta dificultad, pues las elecciones de los individuos para evaluar comparaciones reales de valor hedónico no son siempre fiables (como en casos de adicción a sustancias, por ejemplo).

Además, en un cierto punto, el sufrimiento puede ser tan insoportable que un individuo podría preferir intercambiar todo placer futuro a cambio de hacer que el dolor se detuviera; del mismo modo, el placer en otro momento dado podría ser tan intenso que este podría aceptar, a cambio de que continuara, una tortura futura (elección de la que luego podría llegar a arrepentirse). Alguien podría resolver la situación considerando las evaluaciones subjetivas del individuo en un estado de calma. Sin embargo, de nuevo, el problema es que la persona que hace una elección en un momento tranquilo no es la misma que la persona cuyo juicio está distorsionado por un episodio de tortura o de gran placer.

Asimismo, podríamos dar más peso a las declaraciones de quienes han padecido mucho sufrimiento. En este caso, sin embargo, también las personas pueden olvidar la severidad de su sufrimiento pasado al ser incapaces de evocar los sentimientos de lo espantosas que fueron realmente sus vivencias.

Finalmente, merece la pena mencionar que, incluso estando los métodos de medición del dolor en continua investigación, existen varias técnicas que ofrecen datos sobre las distintas intensidades de dolor (Thong et al., 2018; Hjermstad et al., 2011), diversas investigaciones sobre cómo evaluar el dolor que reportan las personas que lo padecen (Jensen et al., 2011), así como estudios comparados de pacientes que sufren grandes cantidades de dolor en situaciones parecidas (Chanques et al., 2010) o en grupos similares de edad (Sánchez-Rodríguez et al., 2012; Birnie et al., 2019). Esto sugiere que, de hecho, sí existen maneras cada vez más efectivas de evaluar el dolor en individuos que lo padecen y que, por tanto, sí hay razones para considerar relevante el estudio de la cuestión de la asimetría entre el dolor y el disfrute.

Si las respuestas a estas objeciones son válidas, podemos considerar que entre las distintas posiciones centradas en el sufrimiento podremos encontrar candidatas firmes a ser axiologías válidas.

\section{CONCLUSIONES}

En este artículo hemos visto que existen razones de mucho peso para defender las axiologías centradas en el sufrimiento. Como vimos, las cuestiones relativas a la creación o no creación de nuevos seres sugieren que existe una asimetría en la manera en que percibimos el sufrimiento con respecto a la felicidad. Asimismo, pueden ofrecerse una serie de argumentos centrados en cómo se comparan los estados positivos y negativos en los que pueden estar los individuos ya existentes que llevan a la misma conclusión. Tales argumentos se ven respaldados por otros que apuntan a la 
preponderancia del sufrimiento sobre el bienestar positivo que de hecho tiene lugar en el mundo, que suponen el hecho de que asumir estas axiologías puede llegar a tener consecuencias desde el punto de vista del valor esperable mucho mejores que las que se podrían esperar de la asunción de puntos de vista simétricos.

Este artículo se ha ocupado de presentar estos argumentos con el único fin de llevar a cabo una defensa de conjunto de las axiologías asimétricas, sin entrar a proponer ningún tipo concreto de esta. Lo cierto es que una gran pluralidad de posiciones podrá aceptar la asimetría, incluyendo a posiciones monistas como el hedonismo negativo y a posiciones pluralistas de lo más variado. Los diferentes argumentos arriba presentados pueden ser empleados en defensa de todas ellas.

Cabe indicar también que este artículo se ha centrado exclusivamente en el campo de la axiología. En el ámbito normativo puede haber otras razones adicionales para aceptar una ética centrada en el sufrimiento. Pero las axiologías que asumen esta perspectiva no nos comprometen con ningún paradigma normativo concreto. Pueden defenderse éticas centradas en el sufrimiento deontológicas, que asuman restricciones normativas al aumento de sufrimiento, consecuencialistas, que constituyen el tipo más representativo de consecuencialismo negativo, o en línea con éticas del carácter centradas en el cuidado de quienes sufren, o que quizás ensalcen la virtud de la compasión y la solidaridad frente a quien sufre. Todas estas diferentes posiciones son compatibles con la aceptación de la asimetría en axiología que aquí se ha defendido.

Todo esto pone de manifiesto que esta perspectiva tiene un gran potencial hasta el momento no suficientemente explorado en teoría del valor y normativa, y que puede tener importantes corolarios prácticos.

\section{Bibliografía}

Algander, P., "A Defence of the Asymmetry in Population Ethics", Res Publica, 18 (2), 2012, pp. 145-157.

Amris, K. et al., "Pain from Torture: Assessment and Management", Pain Reports, 4 (6), 2019, e794.

Animal Ethics, Introduction to Wild Animal Suffering: A Guide to the Issues, Oakland, Animal Ethics, 2020, ht tps://www.animal-ethics.org/introduction-to-wild-animal-suffering/ (consultado el 7-9-2020).

Bastardi, A. et al., "Wishful Thinking: Belief, Desire, and the Motivated Evaluation of Scientific Evidence", Psychological Science, 22 (6), 2011, pp. 731-732.

Baumeister, R.F. et al., "Bad is Stronger than Good",Review of General Psychology, 5 (4), 2001, pp. 323-370.

Benatar, D., Better Never to Have Been: The Harm of Coming into Existence, Oxford, New York, Clarendon Press Oxford University Press, 2006.

Birnie, K.A., et al., "Recommendations for Selection of Self-Report Pain Intensity Measures in Children and Adolescents: A Systematic Review and Quality Assessment of Measurement Properties", PAIN, 160 (1), 2019, pp. 5-18.

Chanques, G. et al., "The Measurement of Pain in Intensive Care Unit: Comparison of 5 Self-Report Intensity Scales", PAIN@, 151 (3), 2010, pp. 711-721.

Derbyshire, S.W.G., "Pain and the Dangers of Objectivity", en S.W.G. Derbyshire (ed.), Meanings of Pain, Cham, Springer, 2016, pp. 23-36.

Diener, E. et al., "Beyond the Hedonic Treadmill: Revising the Adaptation Theory of Well-Being", American Psychologist, 61, 2006, pp. 103-118.

Elstein, D. J., “The Asymmetry of Creating and Not Creating Life”, The Journal of Value Inquiry, 39 (1), 2005, pp. 49-59. 
FAO - Food and Agriculture Organization of the United Nations, "Livestock primary", FAO Statistical Database, 2020, http://faostat.fao.org/site/569/default.aspx\#ancor (consultado el 1-6-2020).

Faria, C. y Paez, E., "Animals in Need: The Problem of Wild Animal Suffering and Intervention in Nature", Relations: Beyond Anthropocentrism, 3, 2015, pp. 7-13.

Faria, C. et al., Animal Ethics Goes Wild: The Problem of Wild Animal Suffering and Intervention in Nature (tesis doctoral), Barcelona, Universitat Pompeu Fabra, 2016.

Frick, J. D., 'Making People Happy, Not Making Happy People': A Defense of the Asymmetry Intuition in Population Ethics (tesis doctoral), Cambridge, Massachusetts, Harvard University, 2014.

Future of Life Institute, "The Future of AI - What Do You Think?", Future of Life Institute, 2017, futureoflife.org/superintelligence-survey/ (consultado el 18-5-2020).

Gardner, M., "A Harm-Based Solution to the Non-Identity Problem”, Ergo, 2, 2015a, pp. 427-444.

Gardner, M., "On the Strength of the Reason Against Harming”, Journal of Moral Philosophy, 2015b, pp. 1-15.

Giordano, J. et al., "Pain Assessment: Subjectivity, Objectivity, and the Use of Neurotechnology Part One: Practical and Ethical Issues", Pain Physician, 13 (4), 2010, pp. 305-315.

Gloor, L., "Tranquilism", Center on Long-Term Risk, 2017, https://longtermrisk.org/tranquilism (consultado el 7-2-2020).

Gloor, L., "The Case for Suffering-Focused Ethics", Center on Long-Term Risk, 2019, https://longtermrisk.org /the-case-for-suffering-focused-ethics (consultado el 3-3-2020).

Grill, K., "Asymmetric Population Axiology: Deliberative Neutrality Delivered”, Philosophical Studies, 174 (1), 2017, pp. 219-236.

Hjermstad, M., et al., "Studies Comparing Numerical Rating Scales, Verbal Rating Scales, and Visual Analogue Scales for Assessment of Pain Intensity in Adults: A Systematic Literature Review", Journal of Pain and Symptom Management, 41 (6), 2011, pp. 1073-1093.

Horta, O., "Animal Suffering in Nature: The Case for Intervention", Environmental Ethics, 39 (3), 2017, pp. 261-279.

Hurka, T., “Asymmetries in Value”, Noûs, 44, 2010, pp. 199-223.

Jensen, M.P. y Karoly, P., "Self-Report Scales and Procedures for Assessing Pain in Adults”, en D.C. Turk y R. Melzack (eds.), Handbook of Pain Assessment, Nueva York, The Guilford Press, 2011, pp. 19-44.

Mathison, E., Asymmetries and Ill-Being (tesis doctoral), Toronto, Ontario, University of Toronto, 2018.

Mayerfeld, J., "The Moral Asymmetry of Happiness and Suffering”, The Southern Journal of Philosophy, 34 (3), 1996, pp. 317-338.

Mayerfeld, J., Suffering and Moral Responsibility, New York/Oxford, Oxford University Press, 1999.

Meacham, C.J.G., "Person-Affecting Views and Saturating Counterpart Relations", Philosophical Studies, 158 (2), 2012, pp. 257-87.

Minsky, M., The Emotion Machine: Commonsense Thinking, Artificial Intelligence, and the Future of the Human Mind, New York, Simon \& Schuster, 2006.

Mood, A. y Brooke, P., "Estimating the Number of Farmed Fish Killed in Global Aquaculture Each Year", Fishcount, 2012, http://fishcount.org.uk/published/std/fishcountstudy2.pdf (consultado el 1-6-2020).

Narveson, J., "Future People and Us", en R.I. Sikora, y B. Barry (eds.), Obligations to Future Generations, Philadelphia, Temple University Press, 1978, pp. 38-60.

Ord, T., “Moral Trade”, Ethics, 126, 2015, pp. 118-138.

Ratnam, M.V.R.K. y Rao, D.B., Dukkha: Suffering in Early Buddhism, New Delhi, Discovery Publishing House, 2003. 
Roberts, M.A., “An Asymmetry in the Ethics of Procreation”, Philosophy Compass, 6 (11), 2011a, pp. 765-776. Roberts, M. A., “The Asymmetry: A Solution”, Theoria, 77 (4), 2011b, pp. 333-367.

Rozin, P. y Royzman, E.B., "Negativity Bias, Negativity Dominance, and Contagion", Personality and Social Psychology Review, 5 (4), 2001, pp. 296-320.

Ryder, R. D., "Painism: Ethics, Animal Rights and Environmentalism”, Global Bioethics, 5 (4), 1992, pp. 27-35.

Ryder, R. D., "Painism: Some Moral Rules for the Civilized Experimenter", Cambridge Quarterly of Healthcare Ethics, 1999 (8), pp. 35-42.

Ryder, R. D., Painism: A Modern Morality, Open Gate Press, 2003.

Ryder, R. D., "All Beings that Feel Pain Deserve Human Rights", The Guardian, 2005, https://www.theguardi an.com/uk/2005/aug/06/animalwelfare (consultado el 15-03-2021).

Sánchez-Rodríguez, E. et al., "A Comparison of Four Self-Report Scales of Pain Intensity in 6-to 8-Year-Old Children", PAIN@, 153 (8), 2012, pp. 1715-1719.

Schopenhauer, A., Sobre la voluntad de la naturaleza, Madrid, Alianza, 1970.

Schopenhauer, A., El amor, las mujeres y la muerte, Madrid, EDAF, 1989.

Schopenhauer, A., Los designios del destino, Madrid, Tecnos, 1994.

Schopenhauer, A., Una fantasía metafísica, Córdoba, Alción, 1995.

Schopenhauer, A., Sobre el dolor del mundo, el suicidio y la voluntad de vivir, Madrid, Tecnos, 1999.

Schopenhauer, A., El mundo como voluntad y representación, Madrid, Akal, 2005.

Singer, P., Ética práctica, Barcelona, Ariel, 1991.

Singer, P., Animal Liberation, London, Random House, 1995.

Singer, P., The Life You Can Save. How to Do Your Part to End World Poverty, New York, Random House Trade Paperbacks, 2010.

Smith, A., The Theory of Moral Sentiments, Oxford, Oxford University Press, 1759.

Steinbock, B. y McClamrock, R., "When is Birth Unfair to the Child?", Hastings Center Report, 24, 1994, pp. 15-21.

Thong, I.S.K. et al., "The Validity of Pain Intensity Measures: What Do the NRS, VAS, VRS, and FPS-R Measure?", Scandinavian Journal of Pain, 18 (1), 2018, pp. 99-107.

Tomasik, B., “The Importance of Wild-Animal Suffering”, Relations: Beyond Anthropocentrism, 3, 2015, pp. 133-152.

Tooley, M., "Value, Obligation and the Asymmetry Question”, Bioethics, 12 (2), 1998, pp. 111-124.

Unger, P., Living High and Letting Die. Our Illusion of Innocence, New York, Oxford University Press, 1996.

Vinding, M., Suffering-Focused Ethics: Defense and Implications, Copenhagen, Ratio Ethica, 2020.

Notas

$1 \quad$ Singer (1991) discute el caso de las vidas que no valen la pena y pueden considerarse peor que la existencia. En este sentido, ver también Benatar (2006) y Steinbock y McClamrock (1994).

2 Esta asimetría se da no tanto si pensamos en cambios a mejor como en cambios a peor en la situación. Igual que entendemos que reducir el sufrimiento extremo es mejor que crear disfrute extremo, entendemos que aumentar el sufrimiento de moderado a extremo es peor que pasar de un placer extremo a uno moderado.

3 Sin embargo, no sucede así en casos opuestos. Las personas que han sido sometidas a tortura extrema muestran síntomas de trauma psicológico y físico, incluyendo dolor crónico, tiempo después de haber sufrido dicha tortura (Amris et al., 2019). 
4 En su filosofía pesimista, Schopenhauer ofrece una fundamentación ontológica de esta situación generalizada de no-felicidad. A nivel de antropología filosófica, describe al ser humano como inherentemente cruel y egoísta. A nivel ontológico, presenta la noción de voluntad, que es un querer, un impulso, un ímpetu ciego e irracional. Este principio energético es para Schopenhauer la única realidad, mientras que todo lo demás es mera apariencia, representación. La única manera de sobrellevar la carga que supone la vida es la negación de la voluntad de querer mediante la razón, que conduce a un estado de ataraxia o nirvana (Schopenhauer, 1970; Schopenhauer, 1989; Schopenhauer, 1994; Schopenhauer, 1995; Schopenhauer, 1999; Schopenhauer, 2005).

5 Estas intuiciones son sostenidas también desde posiciones budistas (Ratnam y Rao, 2003).

6 Una consecuencia de esta visión consiste en el "comercio moral" (Ord, 2015), que se refiere a que personas con distintos puntos de vista morales pueden intercambiar bienes o servicios tal que ambas partes sientan que el mundo mejoró o que sus obligaciones morales fueron satisfechas. 